\title{
A POCOS KILÓMETROS, PERO EN OTRO PAÍS. EL RETIRO DE JUBILADOS ESTADOUNIDENSES EN BAJA CALIFORNIA, MÉXICO ${ }^{1}$
}

\author{
Raúl Lardiés Bosque \\ Dpto. de Geografía y Ordenación del Territorio, Universidad de Zaragoza \\ rlardies@unizar.es
}

\begin{abstract}
Resumen: México se ha convertido en el principal país de Iberoamérica en recibir jubilados procedentes de Estados Unidos. Este colectivo de inmigrantes se ha extendido por varios enclaves del país, pero una importante comunidad se localiza en la zona fronteriza (en el corredor 'Ensenada-Tijuana', estado de Baja California). Este artículo pretende conocer las razones que influyen en el traslado residencial de los jubilados a esta zona de México, y se basa en la realización de 29 entrevistas en profundidad. Los resultados apuntan a que, sobre todo, factores ambientales y también socioeconómicos determinan el traslado; en concreto, el buen clima, las diferencias de precios y de nivel de vida entre ambos países, además de otras razones como la cercanía y el desarrollo de un entorno cultural y lingüístico propicio, favorecen la movilidad residencial de este colectivo de personas.
\end{abstract}

Palabras clave: retiro, jubilados, movilidad residencial, México, Estados Unidos.

\begin{abstract}
Mexico has become the Latin American country which receives the largest number of retired people coming from the USA. This group of immigrants has spread to several places in the country, but an
\end{abstract}

Recibido: 7-12-10. Aceptado: 22-11-11

${ }^{1}$ Parte de esta investigación se llevó a cabo en El Colegio de la Frontera Norte (COLEF, Tijuana-México) durante junio y julio de 2009 y estuvo cofinanciada por la Secretaría de Relaciones Exteriores (SRE) del Gobierno de México y por la Junta de Extremadura. El autor agradece al Dr. Roberto.Ham y a Eva Palacios, ambos del Departamento de Estudios de Población del COLEF, la ayuda prestada durante el trabajo de campo. Tampoco olvida a la Lcda. Marisa Molina, responsable de la FRAO (Foreign Resident Attention Office) en Playas de Rosarito (estado de Baja California), por su colaboración en la investigación, ni a todos los jubilados estadounidenses entrevistados, por su dedicación de tiempo y amabilidad. El autor está igualmente en deuda con los dos evaluadores anónimos por las sugerencias hechas para mejorar el artículo. 
important community is located in the border area (in the corridor 'Ensenada-Tijuana', state of Baja California). This article seeks to know the reasons that affect the residential relocation of retirees moving to this area of Mexico, and is based on the completion of 29 in deep-interviews. The results suggest that environmental and socioeconomic factors mainly determine the changes; in particular, the good climate, the differences in prices and living standards between both countries promote the residential mobility of this group of people; other reasons such as the proximity and the development of a particular cultural and linguistic environment are also important.

Keywords: retirement, retired, residencial mobility, Mexico, United States.

\section{Introducción}

La importancia de México como país de retiro de jubilados extranjeros está aumentando en las últimas décadas (Lizárraga, 2008), y también la de este fenómeno como tema de estudio, pero no por ser país de origen de esos desplazamientos, sino de destino de inmigrantes jubilados (Coates et al., 2002).

A pesar de la juventud de esta modalidad migratoria, los estudios realizados hasta el momento han intentado cuantificar las migraciones de jubilados desde EE.UU. y Canadá hacia México, delimitando los principales lugares de destino y las principales características demográficas de los migrantes jubilados, las motivaciones de los desplazamientos (incluso desde perspectivas más psico-antropológicas y de identidad como Balslev y Velázquez, 2011), las formas de vida y comportamientos de esta población (Coates et al., 2002; Kiy y McEnany, 2010a; Sunil et al., 2007); más recientemente, el interés se ha puesto en las cuestiones de salud y de asistencia médica (Kiy y McEnany, 2010b), en la vivienda (Kiy y McEnany, 2010c), en su integración (Banks, 2009; Lizárraga, 2006), y en su participación en actividades de voluntariado y de caridad (Kiy y McEnany, 2010d). El enfoque de lo transnacional, tan extendido en estudios de carácter migratorio (O'Reilly, 2000), y en particular entre las migraciones internacionales de jubilados (Gustafson, 2001), también se ha aplicado a los flujos de personas mayores que se trasladan a México (Lizárraga, 2008).

Con este artículo se intenta seguir analizando el comportamiento migratorio de este colectivo, prestando atención a los jubilados residentes en dos de los cinco municipios (Playas de Rosarito y Tijuana) que integran la península de Baja California, a escasos kilómetros de la frontera con EE.UU. El interés radica en conocer las razones 
que desatan esta forma de movilidad residencial hacia México, lo cual constituirá el paso previo para poder explicar muchos de los comportamientos de este colectivo, su integración, su movilidad espacial y los contactos que mantienen entre el país de origen y el elegido como destino.

El trabajo de campo se realizó en la zona fronteriza de Tijuana con EE.UU. (estado de Baja California; mapas 1, 2 y 3) durante junio y julio de 2009. La metodología utilizada ha sido cualitativa y se basa en 29 entrevistas en profundidad realizadas a residentes jubilados de origen estadounidense que residen en esta parte de México. Las entrevistas fueron realizadas y grabadas en inglés (idioma nativo de los entrevistados) con ayuda de una grabadora digital, tuvieron una duración de entre cuarenta minutos y algo más de una hora, y posteriormente fueron transcritas. La mayoría se realizaron en la localidad de Playas de Rosarito (a $30 \mathrm{kms}$. de la frontera de Tijuana con EE.UU.), aunque algunas también en el núcleo de San Antonio del Mar, por tratarse de una zona residencial perteneciente al municipio de Tijuana, muy próxima a Playas. Esta zona costera del estado de Baja California forma parte del corredor entre Ensenada hasta la frontera con EE.UU., y en ella se han desarrollado numerosos complejos residenciales con vistas al mar especialmente destinados a jubilados (Fotografías 1 y 2).

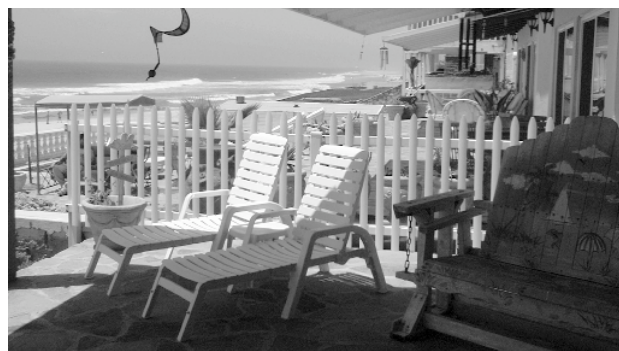

Fotografía 1. Vivienda de una jubilada en una urbanización de Playas de Rosarito, México, 2009. Fotografía del autor.

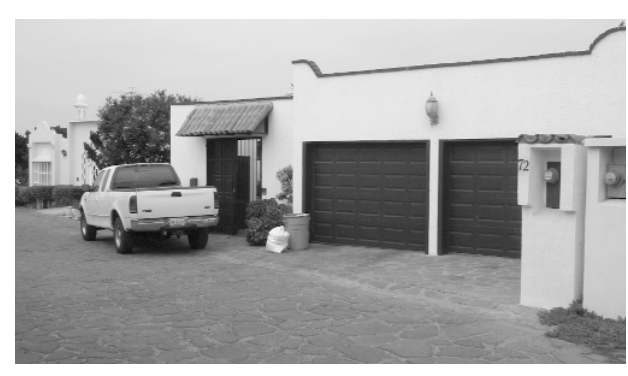

Fotografía 2. Vivienda de un jubilado en una urbanización de Playas de Rosarito, México, 2009. Fotografía el autor.

\section{México, país de destino de jubilados}

México se ha convertido en un destino muy popular para el colectivo de jubilados estadounidenses, y en el país donde se retira el mayor número, entre quienes deciden pasar su jubilación fuera de su país (Michael, 1997; Shelley, 1996). Algunos incentivos de tipo económico, pero también político, climático y sociocultural, unido a factores ambientales y de tipo natural, tienen un efecto decisivo en estos traslados 
residenciales de jubilados, sin olvidar la cercanía de ambos países (Sunil et al., 2007) y la oferta asistencial y sanitaria.

El aumento de la movilidad residencial de jubilados estadounidenses a diversos países iberoamericanos, y en concreto a México, se puede explicar también a partir de las implicaciones demográficas que está teniendo el aumento de los efectivos pertenecientes a la generación del baby boom en EE.UU.; más de 70 millones de personas pertenecen a esa generación (nacidos entre 1946 y 1964), de los que más de 65 millones están todavía con vida (Rogerson y Kim, 2005). En concreto, y según el último censo de población de EE.UU. (U.S. Census Bureau, 2000a), la población con 65 y más años era de 38 millones en el año 2000 y de alrededor de 40 millones en 2010, aunque se estima que sea de 88,5 millones en 2050 (U.S. Census Bureau, 2000b); ante tal crecimiento, la población de ese grupo de edad pasará de representar el $12,9 \%$ del total en 2010 a ser el $20,2 \%$ en 2050.

Se trata, por lo tanto, de un colectivo de población de gran interés y de elevado potencial económico para el gobierno mexicano (Secretaría de Turismo, 2000), ya que pocas generaciones anteriores habían contado con un poder adquisitivo como del que dispone la actual, y pocos mayores habían tenido tantas posibilidades de viajar y salir al extranjero.

\section{2. ¿Cuántos son y dónde están los inmigrantes jubilados?}

México era el país de Centro y Sudamérica donde residía en 1999 el mayor número de ciudadanos estadounidenses (1.036.300 y el 80,4\% de los emigrados) (MPI, 2006), aunque diversos organismos discrepan sobre el número. En cualquier caso, la llegada de jubilados ha sido progresiva en las últimas décadas y se ha producido en paralelo al aumento de la población total extranjera en México, que ha crecido notablemente entre 1970 y el año 2010 (Tabla 1); según el Instituto de

Tabla 1. Evolución de los extranjeros en México, 1970-2010.

\begin{tabular}{|cccccc|}
\hline Años & Población total & Extranjeros & $\%$ & $\begin{array}{c}\text { Extranjeros } \\
\text { de EUA }\end{array}$ & $\begin{array}{c}\text { \% de estadounidenses/ } \\
\text { extranjeros }\end{array}$ \\
\hline 1970 & 48.225 .238 & 191.159 & 0,40 & 97.229 & 50,9 \\
1980 & 66.846 .833 & 268.900 & 0,40 & 157.117 & 58,4 \\
1990 & 81.249 .645 & 340.824 & 0,42 & 194.619 & 57,1 \\
2000 & 97.014 .732 & 492.617 & 0,51 & 358.614 & 69,0 \\
2010 & 112.336 .538 & 961.121 & 0,85 & 738.103 & 76,8 \\
\hline
\end{tabular}


Migraciones estadounidense (MPI, 2006), un gran crecimiento de esa población se detectó entre 1970 y 1980 (57,6\%), y en concreto entre 1980 y 1990 (84,3\%).

Las pautas de localización de los jubilados de origen estadounidense difieren notablemente de las desarrolladas por el total de la población de origen extranjero, por lo que no hay competencia entre las localidades que acogen a un tipo y otro de inmigrantes. Donde mejor se aprecian las distintas pautas de asentamiento es a escala local, ya que estos jubilados se concentran básicamente en localidades de tamaño demográfico pequeño o mediano, más en zonas costeras, y menos de interior, aunque sí cerca de lagos.

También hay diferencias de localización por estados; por ejemplo, en el mapa 2 (en el que se refleja el peso de esa población mayor sobre el total de los extranjeros) se puede apreciar la concentración de jubilados de origen extranjero en determinados estados costeros; por el contrario, el total de la población extranjera (mapa 1) no parece seguir el mismo patrón de asentamiento, diluyéndose ésta de forma mucho más homogénea y, sobre todo, concentrándose en los estados fronterizos y en aquellos que albergan grandes zonas urbanas.

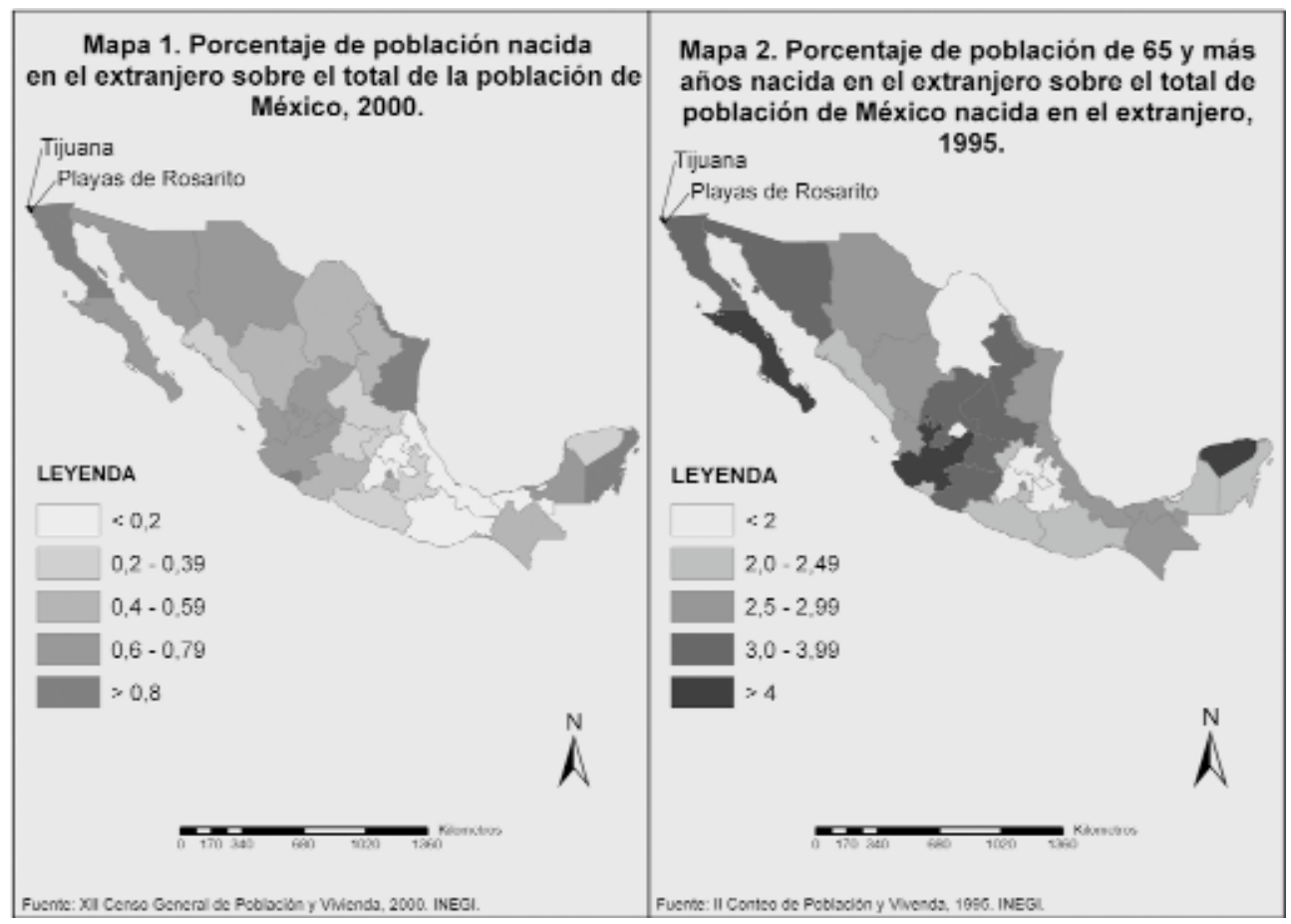


La distribución espacial de los jubilados de origen estadounidense en México permite detectar cierta "segregación" residencial. El mayor número se encuentra en localidades de la costa del Pacífico (corredor norte de la Baja California, y también en Los Cabos, en Baja California Sur) y del centro del país, sobre todo alrededor del Lago Chapala (estado de Jalisco) y San Miguel de Allende, además de en algunas zonas de Yucatán (Sunil et al., 2007) (mapas 2 y 3). En concreto, los alrededores del lago Chapala son destino importante de un gran volumen de población bohemia y artistas, y allí se concentra una numerosa colonia de jubilados estadounidenses y también de canadienses; al contrario, los jubilados que se trasladan a Los Cabos (el mayor destino de jubilados en Baja California Sur) destacan por su elevado nivel adquisitivo, muy superior, por lo general, a quienes residen en el corredor Tijuana-Ensenada de la Baja California. En cualquier caso, y según el último censo de población realizado en México (2000), el número de retirados extranjeros de origen estadounidense es muy elevado en los estados del norte del país; concretamente en Baja California su número creció entre 1990 y 2000 un 43,5\%, mientras que en la Baja California Sur lo hizo un $188 \%$.

Mapa 3. Estados en México.

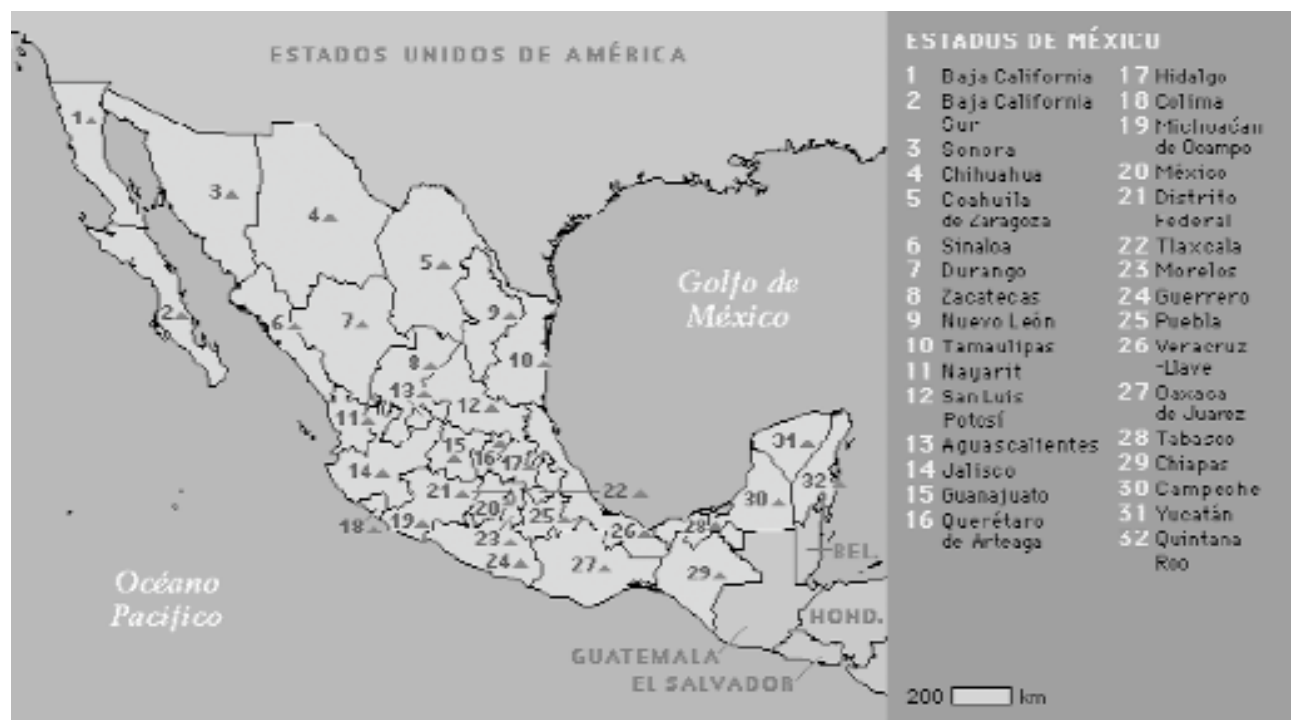

Tomado de: http://www.grito-independencia-mexico.com 


\section{3. ¿Por qué elegir México como país donde pasar la jubilación?}

El mundo actual se caracteriza por una movilidad espacial no comparable con la del pasado, que no se sabe si es hija o madre de la globalización, pero que se ha integrado en la vida cotidiana a partir de la modificación de los imaginarios (Hiernaux, 2006). El aumento de la movilidad, expresada en una nueva forma de libertad, actúa de "telón de fondo" causal en cualquier proceso de movilidad residencial o migración, aunque en el caso de las migraciones (internacionales) de jubilados, las favorecen también otros factores como el aumento de la esperanza de vida, la salida prematura del mercado de trabajo de mucha población (prejubilados), y la mayor disponibilidad de ingresos que otros grupos de población (Casado-Díaz, 2006); además, estas personas están libres de obligaciones laborales y, generalmente, familiares (o no tienen hijos, o los tienen ya crecidos, y sus padres ya han fallecido, por lo que no tienen personas dependientes a quien cuidar) (Bell y Ward, 2000).

La acumulación de experiencias vitales gracias a los viajes realizados y a los lugares turísticos visitados a lo largo de la vida es otro factor importante que predispone a la movilidad, por cuanto se genera un fuerte nexo entre turismo y migración (Casado-Díaz, 2006). La familiaridad que otorgan las reiteradas visitas turísticas realizadas periódicamente a un destino en vacaciones favorece que posteriormente sean elegidos como lugares a donde trasladar el lugar de residencia.

En este sentido, la población estadounidense ha desarrollado tradicionalmente sobre México todo un imaginario de individualidad y tranquilidad, que ha devenido en un modelo de turismo residencial caracterizado por el consumismo y la masificación (Hiernaux, 2009). De esa forma, las zonas tradicionalmente turísticas han aumentado su oferta residencial, al socaire de la modernización del sector de la construcción y de la presencia de los promotores inmobiliarios en la producción de segundas residencias (Hiernaux, 2005)

En el caso del 'cordón costero' comprendido entre Ensenada y la frontera, la expansión del turismo residencial ha permitido durante décadas a muchos norteamericanos adquirir una vivienda para vacaciones, o pasar intermitentes temporadas como turistas, en buena medida por la proximidad con EE.UU. Ya en los años 60 la prensa de San Diego (The San Diego Union, 1968) señalaba que unos 10 millones de estadounidenses visitaban la zona de Tijuana por motivos turísticos, destacando las ventajas de adquirir alguna propiedad en la zona fronteriza. De esta forma, la confianza que los norteamericanos han mostrado en esta zona como lugar de vacaciones ha permitido crear un enorme mercado inmobiliario y de residencias secundarias, de forma similar a como ha ocurrido en otras partes del país (Truly, 2002).

Pero al margen de estos factores de tipo general, cuando se pregunta concretamente a los jubilados por sus traslados residenciales, éstos suelen estar motivados por 
cuestiones y oportunidades económicas, pero también por los beneficios que se prevé vayan a obtenerse en relación a la mejora de su calidad de vida y bienestar. Se suelen sentir atraídos por zonas con climas benignos y cálidos, y con amenidades y oportunidades de ocio; también influye que el destino residencial se conociera debido a experiencias turísticas previas y por haber pasado vacaciones en él, bien alojados en hoteles, casas de amigos o familiares, o incluso en una residencia secundaria propia. De esta forma, algo que comienza como desplazamientos temporales durante periodos de vacaciones cortos (o largos), se acaba convirtiendo en una migración 'permanente'.

\subsection{Factores físico-ambientales y económicos}

Muchos de los factores anteriores se han identificado en los desplazamientos de jubilados estadounidenses a México (Kiy y McEnany, 2010d), aunque se dan con particularidades y matices propios. Los de tipo ambiental se han destacado como los más importantes (Coates et al., 2002; Melton, 1997; Shelley, 1996), si bien están muy relacionados (por lo que existe gran dificultad para aislarlos) con el deseo de mejorar su calidad de vida, ganar tranquilidad, con vivir en un clima agradable, y si es posible cerca del mar (Kiy y McEnany, 2010a; MPI, 2006):

I really enjoy Baja, the ocean. There's no where in California where I can afford to live on the ocean unless I had 3 million dollars to have a nice home on the ocean. (Hombre, 69 años, casado, nacido en California).

Entre las ventajas con que cuentan las localidades de Baja California para el retiro se encuentra la menor humedad ambiental, comparada con la de otros lugares del interior de México, o incluso con la de otros países que pueden ser competencia (Panamá, Costa Rica, etc.), debido a que el clima seco es beneficioso para muchos procesos reumáticos y de huesos entre la población mayor. En el siguiente testimonio, una jubilada considera los beneficios del clima para su enfermedad, y se refiere a la búsqueda que hizo de un lugar tranquilo y pacífico donde jubilarse, cerca del mar:

With my disease, the humidity is very bad [...]. That's why I don't go to San Felipe a lot because with multiple sclerosis the humidity and the heat, you have to be very, very careful and not step over a certain line. But yes, I did consider farther south. In fact, a few years ago when this started building up, a friend and I said, "you know, let's take a big trip and see if by the ocean we can find, not necessarily cheaper but quieter, peaceful, just like Rosarito used to be". (Mujer, 75 años, separada, nacida en Illinois).

Los destinos costeros de Baja California tienen un gran poder de atracción y de evocación como lugares de relajación y de descanso, y se conocían antes del traslado 
por haber sido lugares de repetidos viajes y visitas. La mujer del testimonio anterior alude también a la mayor calidad de vida que puede disfrutar ahora en comparación con su lugar previo de residencia en EE.UU., etapa que la asocia al tragajo y al estrés:

As far as the quality of life, just before I retired early and came here, there were many, many, many stresses [...]. Where I lived [...] it was small but there were many, many, stresses. So when I came here, every time I would come at night and you'd see the old Tijuana, with the lights; and I'd get there and I'd think "I'm coming home". We didn't have the house yet but it just was home. Because my happiest years, really, of my life were the years in "el mar", the beach. So it was like coming home [...]. You know, I'm willing to sit in the line but that's the basic thing. (Mujer, 75 años, separada, nacida en Illinois).

Es difícil desvincular la atracción climática de otros factores como los económicos, debido a la fuerte influencia que tienen las diferencias de precios y de nivel de vida entre ambos países; esas diferencia han permitido a muchos jubilados poder adquirir la casa de sus sueños al borde del mar, algo que es impensable para la mayoría de la población en EE.UU. (Kiy y McEnany, 2010c), y sobre todo en California, de donde proceden muchos de los jubilados entrevistados. Los menores precios en México les permite también disponer de algunos servicios que en su país no se podrían costear (servicio doméstico y jardinero, pero también vigilancia y seguridad particular entre quienes viven en una urbanización privada):

Nunca había tenido sirvienta y aquí se puede conseguir alguien por..., mucho más económico. Y nunca habría tenido los fondos para poder vivir frente al mar. Parece como si fuese millonaria, y no soy millonaria. (Mujer, 78 años, divorciada, nacida en París, y residente en varios estados de EE.UU. la mayor parte de su vida).

La ventaja económica de vivir en México se manifiesta principalmente en el acceso a una vivienda más barata, pero, sobre todo, localizada al borde del mar:

Economically, just living in the State of California and having to pay state income tax is a disadvantage for retirement. We were of the opinion that you could make your dollar stretch so much further being here. Either way would have worked out fine except here it affords us the availability to go travel a lot more than we would have been able to in the States. And frankly even though we lived in a very nice home and a nice location in the States, living here were we are with the view we have and near the ocean is something that we never could have afforded in San Diego, and yet here is just so wonderful to be here were we actually live. Economically it just makes it a great benefit to be here. (Hombre, 60 años, casado, nacido en Oklahoma).

Indirectamente, también la movilidad residencial se ha convertido en una estrategia para rentabilizar inversiones o los ingresos disponibles (Hiernaux, 2009). Por ejemplo, otro entrevistado reconoce que haberse trasladado a vivir a México le ha permitido vender una vivienda en California, en donde el jardín era demasiado grande para poder cuidarlo él como venía haciendo, y acceder a otra vivienda en 
México con un jardín más pequeño del que se puede ocupar él mismo y que exige menos trabajo:

When I was younger, my garden was very big and I took care of it myself [...]. I had the energy to take care of such a big garden but when I got near my 70's..., I'm 73 years-old now. When I got to be older it was very hard to take care of [...]. I couldn't do it anymore [...]. When I couldn't take care of suck garden I decided what I want is a small garden, you see what I have here. A garden that I can work at, a half an hour every day is enough to take care of this garden. (Hombre, 73 años, casado, nacido en Toronto, Canadá).

Sin embargo, tanto si sólo se tiene en cuenta el clima, como otros factores atrayentes hacia México, hay que considerar la importante competencia que sufre el país como lugar internacional de retiro para los estadounidenses. Parecidas condiciones climáticas y económicas pueden darse en países iberoamericanos, aunque la ventaja de la cercanía a EE.UU. hace determinante el desplazamiento a México. La siguiente mujer manifiesta cómo comenzó con su marido a alquilar viviendas en esta zona, cómo consideró retirarse en otros países, y cómo decidieron, finalmente, establecerse en Baja California.

That was in 1996. So we would rent houses sporadically and come and spend weekends to see if we liked it and if we were comfortable there. We thought about retiring possibly to Ireland or Hawai, Phoenix, Arizona area for the temperature, the climate. Not Ireland, that was because we liked Ireland. So after we rented places for about a year and a half. My husband decided, and I agreed that we would buy a retirement home here, and we did eventually. We spent time back and forth. Originally, we would spend six months in Las Vegas and six months here because the temperature in Las Vegas in the summer time is too hot, and I didn't like that. (Mujer, 60 años, casada, nacida en Pennsylvania).

Por lo tanto, los entrevistados suelen destacar la cercanía a EE.UU. como una de las razones más importantes del traslado, ya que poder vivir en la zona fronteriza es importante para seguir disfrutando de las ventajas de ambos países, incluyendo que la mayoría de la gente en esta parte de México hable inglés.

\subsection{Factores socio-sanitarios}

Otros factores también aludidos que justifican el cambio residencial son los de tipo sociocultural y, en concreto, los relacionados con la atracción de los jubilados por la cultura y por la forma de vida mexicana, pero también con el gran capital social generado en muchos lugares de México. A pesar de la idealización de México como un país peligroso, donde la gente es fanática, perezosa y poco fiable, al mismo tiempo persiste una fascinación por muchos aspectos de la cultura mexicana (Balslev y Velázquez, 2011). 
Eso ha influido para que en la zona fronteriza se hayan desarrollado comunidades anglófonas de jubilados que han ido creciendo a partir del uso generalizado del inglés y también de la expansión de hábitos culturales y costumbres importadas del país vecino, de la misma forma que ha ocurrido en otras comunidades de jubilados (Rodríguez et al., 2004). Tal "caldo de cultivo" ha permitido el desarrollo de numerosas asociaciones y grupos, y el mantenimiento de redes y prácticas transnacionales entre los estadounidenses residentes en México y en el país de origen (Lizárraga, 2006; Lizárraga, 2008). Administrativamente, estos jubilados residen en un país distinto al suyo, aunque en el terreno de lo privado no tienen la sensación de estar viviendo en otro país, básicamente por la posibilidad de que en estos destinos fronterizos se les brinda la posibilidad de sentirse "como en casa" y en un entorno cultural no muy diferente al de su país de origen; tales experiencias son posibles gracias a poder hablar habitualmente inglés entre ellos y también con la población de origen mexicano, reunirse, organizar y compartir sus actividades diarias con personas procedentes de su mismo país, y hasta poder continuar con un régimen frecuente de visitas a EE.UU. para atender sus compras, ir al médico, o reunirse con amigos y/o familia:

About half my friends are Americans or Canadians, the other half are locals. (Mujer, 59 años, casada por segunda vez, nacida en Michigan).

Even the mayor with a room full of Mexican citizens will conduct the entire meeting in English because they all speak it [...].

If you come into Rosarito and you go in a restaurant and sit down and you try to use your Spanish, they'll speak to you in English because they want to use their English. It was funny when we first came down we'd start out in Spanish and they'd say English, we'd say Spanish, they'd say English. (Hombre, 60 años, casado, nacido en Oklahoma).

En la toma de decisión para establecer el nuevo lugar de residencia en esta zona fronteriza hay otro factor importante que es el sanitario, relacionado con la oferta médica que ofrece México y con la posibilidad de seguir disfrutando de la cobertura médica (Medicare) que ofrece la jubilación en EE.UU. (Dibble, 2010; Kiy y McEnany, 2010b). La proximidad a EE.UU., pero también el acceso a cuidados médicos y a medicinas relativamente baratos en México, sobre todo en comparación con lo que cuestan en EE.UU., se convierte para la mayoría de los entrevistados en un factor determinante del traslado; según estimaciones del gobierno mexicano, el coste de los tratamientos sanitarios en México es alrededor de un 70\% inferior (Oppenheimer, 2010). Además, la abundante oferta de farmacias y para-farmacias arrastra diariamente a miles de ciudadanos norteamericanos hacia la frontera, por lo que se ha estimado en medio millón de estadounidenses los que anualmente la atraviesan y entran en el estado de Baja California para comprar medicinas o para recibir tratamientos médicos (Dibble, 2010).

I choose Rosarito Beach [...] because as a retiree I get Medicaid, I'm sorry..., I get Social Security which means I get Medicare in the U.S.A. So I want to be close to the border; 
[...] proximity to the border [...]. I came to Rosarito Beach because when I have a medical problem, 20 minutes and I'm in the U.S.A. Otherwise I would be living in Lake Chapala, nice place, or maybe Guadalajara. (Hombre, 85 años, soltero, nacido en Hawai).

En menor medida, también algunos entrevistados han destacado como razones para abandonar EE.UU. las de tipo político, en relación al desacuerdo con la forma en que los políticos han estado gobernando el país.

\section{Conclusiones}

México es el país que más jubilados de origen estadounidense recibe, tendencia que parece ser imparable a medida que los integrantes de las generaciones del baby boom estadounidense se vayan jubilando, y debido también a la cercanía entre ambos países. No hay duda de la importancia que el fenómeno está tomando en México, y de que se trata de un tema cada vez de más actualidad (Lizárraga, 2008).

Hasta el momento se han conocido algunos aspectos de esta movilidad, pero queda pendiente ir introduciendo herramientas de planificación y control para dotar a los lugares de destino de servicios y equipamientos para esta población (servicios de salud, de ocio y otros especializados), previendo el futuro envejecimiento de la población norteamericana y el aumento de baby boomers retirados procedentes (sobre todo) de EE.UU. Esa planificación hará que afloren asuntos de tipo económico como la financiación de recursos, pero también otros de tipo territorial y ambiental ligados a la urbanización excesiva que en ocasiones sufren los destinos, relacionados con las múltiple facetas del turismo residencial, por no hablar de los derechos sociales y políticos de esa nueva población, o de la integración y participación en sus nuevos lugares de residencia.

También entre los aspectos pendientes de analizar está el comportamiento espacial de estos inmigrantes, como la movilidad repetida entre el país de origen y de destino, o incluso hacia terceros, debido a múltiples razones como el ocio, sanitarias, familiares, etc., o la posibilidad de retorno en relación, por ejemplo, a factores como la duración de la estancia, la existencia de familia y su composición, o la distancia y diferencia de nivel de vida entre los lugares de origen y destino.

Igualmente deberían mejorar las estadísticas para hacerlas fiables y certeras, con el fin de poder cuantificar con exactitud y de forma fehaciente la magnitud de estos desplazamientos de población. Existe también el reto de desarrollar modelos teóricos $\mathrm{y}$ aplicados que relacionen esta movilidad con el auge del turismo y con el retiro. 
Todo ello será de gran ayuda para investigadores y planificadores, en aras de la mejor comprensión del fenómeno, y para planificar las infraestructuras y dotaciones necesarias en los destinos. Ello pasa necesariamente por el contacto entre disciplinas, por lo que se impone la interdisciplinariedad para el análisis de un fenómeno que no entiende de barreras ni de fronteras. 


\section{Bibliografía}

Balslev Clausen, H. y Velázquez García, M. A. (2011). En búsqueda del México auténtico. Las comunidades norteamericanas en ciudades turísticas de México. En Mazón, T., Huerte, R. y Mantecón, A. (eds.) Construir una nueva vida. Los espacios del turismo y la migración residencial, Santander, Milrazones, pp. 61-80.

Banks, S. P. (2009). Intergenerational ties across borders: Grandparenting narratives by expatriate retirees living in Mexico. Journal of Aging Studies, 23, 3, 178-187.

Bell, M. y Ward, G. (2000). Comparing Permanent Migration with Temporary Mobility: An Analysis of Australian Data Tourism Geographies, 2, 1, 87-107.

Casado-Díaz, Ma․ A. (2006). Retiring to Spain: An analysis of differences among North European Nationals. Journal of Ethnic and Migration Studies 32, 8, 1321-1339.

Coates, K. S.; Healy, R. y Morrison, W. R (2002). Tracking the snowbirds: seasonal migration from Canada to the U.S.A. and Mexico. American Review of Canadian Studies, 32, 3, 433-450.

Dibble, S. (2010). "Mexican health care for Americans studied". San Diego UnionTribune, 21 Abril 2010. [http://www.signonsandiego.com/news/2010/apr/21/mex ican-health-care-for-americans-studied]

Gustafson, P. (2001). Retirement migration and transnational lifestyles. Ageing and Society, 21, 4, 371-394.

Hiernaux, D. (2005). La promoción inmobiliaria y el turismo residencial: el caso mexicano. Scripta Nova. Revista electrónica de geografía y ciencias sociales. Barcelona, Universidad de Barcelona, 1 de agosto de 2005, vol. IX, 194 (05). <http:// www.ub.es/geocrit/sn/sn-194-05.htm> (Consulta: 25.10.2011).
Hiernaux, D. (2006). Geografía del turismo. En Lindón, A. y Hiernaux, D. (dirs.) Tratado de Geografía Humana. México, Anthropos-Universidad Autónoma de México, p. 401-432.

Hiernaux, D. (2009). Los imaginarios del turismo residencial: experiencias mexicanas. En Mazón, T., Huete, R. y Mantecón, A. (eds) Turismo, urbanización y estilos de vida. Las nuevas formas de movilidad residencial. Barcelona, Icaria, p. 109-126.

Kiy, R. y Kada, N. (eds.) (2004). Blurred borders. Transboundary Issues and Solutions in the San Diego/Tijuana Border Region. San Diego, International Community Foundation.

Kiy, R. y McEnany, A. (2010a). U.S. Retirement Trends in Mexican Coastal Communities Lifestyle Priorities and Demographics. Report. Marzo. U.S. Retirement in Mexico Research Series. San Diego, International Community Foundation, 17 p.

Kiy, R. y McEnany, A. (2010b). Health Care and Americans Retiring in Mexico. Report. Mayo. U.S. Retirement in Mexico Research Series. San Diego, International Community Foundation, 22 p.

Kiy, R. y McEnany, A. (2010c). Housing and Real Estate Trends among Americans Retiring in Mexico's Coastal Communities. Report. Mayo. U.S. Retirement in Mexico Research Series. San Diego, International Community Foundation, $28 \mathrm{p}$

Kiy, R. y McEnany, A. (2010d). Civic Engagement, Volunteerism and Charitable Giving: Americans Retiring in Mexico's Coastal Communities. Report. Junio. U.S Retirement in Mexico Research Series. San Diego, International Community Foundation, 21 p. 
Lizárraga, O. (2006). Redes sociales e integración social de estadounidenses retirados en Mazatlán. Tesis de Maestría. Culiacán, Universidad Autónoma de Sinaloa.

Lizárraga, O. (2008). La inmigración de jubilados estadounidenses en México y sus prácticas transnacionales. Estudio de caso en Mazatlán, Sinaloa y Cabo San Lucas, Baja California Sur. Migración y Desarrollo, 11, 97-117.

Melton Young Otero, L. (1997). U.S. Retired Persons in Mexico. American Behavioral Scientist, 40, 7, 914-925.

Michael, N. (1997). Living in Mexico. El Paso, Southside Publishing.

MPI (Migration Policy Institute) (2006) America's Emigrants. US Retirement Migration to Mexico and Panama. Washington, DC, The Migration Policy Institute.

Oppenheimer, A. (2010). Mexico hopes to attract U.S. retirees. LJWorld Journal World \& News, 20 abril 2010. [http://www2.ljworld.com/news/2010/apr /20/mexico-hopes-attract-us-retirees].

O'Reilly, K. (2000). The British on the Costa del Sol: Transnational Identities and Local Communities. Londres, Routledge.

Rodríguez, V.; Fernández-Mayoralas, G. y Rojo, F. (2004). International Retirement Migration: Retired Europeans Living on the Costa del Sol, Spain. Population Review, 43, 1, 1-36.

Rogerson, P. A. y Kim, D. (2005). Population distribution and redistribution of the babyboom cohort in the United States: Recent trends and implications. Proceedings of the
National Academy of Sciences of the United States of America, 102, 43, 1531915324

Secretaría de Turismo (2000). Estudio de gran visión del turismo en México: Perspectiva 2020. Reporte analítico. Ciudad de México, Centro de Estudios Superiores en Turismo, Secretaría de Turismo.

Shelley, E. (1996). Your Guide to Retiring to Mexico, Costa Rica and Beyond. New York, Avery Penguin Putnam.

Sunil, T. S.; Rojas, V. y Bradley, D. E. (2007). United States' international retirement migration: the reasons for retiring to the environs of Lake Chapala, Mexico. Ageing E Society, 27, 4, 489-510.

The San Diego Union (1968). New Twists In Old Laws. Lure Americans South Of The Border To Live. The San Diego Union, 23 Julio 1968

Truly, D. (2002). International retirement migration and tourism along the Lake Chapala Riviera: developing a matrix of retirement migration behaviour. Tourism Geographies, 4, 3, 261-281.

U.S. Census Bureau (2000a). Census 2000 Suitland, Maryland, Census Bureau, Population Division.

U.S. Census Bureau (2000b). 2008 National Population Projections (Based on Census 2000). Suitland, Maryland, Census Bureau, Population Division.

Warner, D. C. y Jahnke, L. R. (2001). Toward Better Access to Health Insurance Coverage for U.S. Retirees in Mexico. Salud Pública México, 43, 1, 59-66. 